\title{
The role of VIT1/FBXO11 in the regulation of apoptosis and tyrosinase export from endoplasmic reticulum in cultured melanocytes
}

\author{
CUIPING GUAN ${ }^{1}$, FUQUAN LIN ${ }^{2}$, MIAONI ZHOU ${ }^{1}$, WEISONG HONG ${ }^{1}$, LIFANG FU ${ }^{1}$, \\ WEN XU ${ }^{1}$, DONGYIN LIU ${ }^{1}$, YINSHENG WAN ${ }^{3}$ and AIE XU ${ }^{1}$ \\ ${ }^{1}$ Department of Dermatology, Third People's Hospital of Hangzhou, Hangzhou 310009; \\ ${ }^{2}$ Bioengineering Institute, Zhejiang Sci-Tech University, Hangzhou 310018, P.R. China; \\ ${ }^{3}$ Department of Biology, Providence College, Providence, RI 02918, USA
}

Received January 11, 2010; Accepted March 10, 2010

DOI: 10.3892/ijmm_00000435

\begin{abstract}
Our previous study has shown that VIT1 gene in Chinese vitiligo patients is de facto the FBXO11 gene, and the silencing of that gene has an impact on the ultrastructure of melanocytes. In this study, we further identified the role of the $F B X O 11$ gene in melanocytes and the relationship between dilated endoplasmic reticulum (ER) and tyrosinase by inhibition and overexpression of FBXO11 gene. Cell proliferation, apoptosis, cycle and migration of melanocytes were examined when the FBXO11 gene was silenced or overexpressed. The results showed that $F B X O 11$ gene promoted cell proliferation and suppressed cell apoptosis, and yet had little effect on cell migration. Obvious swelling of ER was found in the cells transfected with siRNA of FBXO11 gene. Interestingly, protein level of tyrosinase was extraordinarily high following inhibition of $\mathrm{FBXO11}$ gene. Further examination revealed that tyrosinase and calreticulin were co-localized in ER of transfected cells following siRNA of $F B X O 11$ gene, suggesting that tyrosinase could not be exported from ER effectively. Collectively, our results support the notion that FBXO11 plays an important role in regulating proliferation and apoptosis of melanocytes, and functional export of tyrosinase from ER in vitiligo melanocytes.
\end{abstract}

\section{Introduction}

Vitiligo is an acquired hypomelanotic disorder characterized by the loss of functional melanocytes from the epidermis.

Correspondence to: Dr Aie Xu, Department of Dermatology, Third People's Hospital of Hangzhou, Hangzhou 310009, P.R. China E-mail: xuaiehz@msn.com

Dr Yinsheng Wan, Department of Biology, Providence College, Providence, RI 02918, USA

E-mail: yswan@providence.edu

Key words: VIT1/FBXO11, melanocyte, endoplasmic reticulum, export, tyrosinase
Large population surveys have shown a prevalence ranging from 0.38 to $1.13 \%$ (1). Why and how melanocytes disappear to induce the characteristic achromic lesions is not fully understood. Current consensus regarding the etiology of vitiligo implies a progressive loss of pigment cells.

Although the pathogenesis of vitiligo cannot be explained by genetics clearly, linkage and association studies have begun to provide strong support for vitiligo susceptibility genes on chromosomes 4q13-q21, 1p31, 7q22, 8p12 and 17p13 (2-4). VIT1, a novel gene, was reportedly to be down-regulated in vitiligo melanocytes (5). Sequence analysis in our previous study revealed that VIT1 gene lacking its intron is de facto the FBXO11 gene (6). FBXO11 belongs to the F-box protein family, which constitutes one subunit of the ubiquitin protein ligase complex called SCF (SKP1-cullin-F-box).

SCF functions in phosphorylation-dependent ubiquitination and induces protein degradation, and the reduced SCF causes dilated endoplasmic reticulum (ER) and results in functional deficiency of key proteins in melanocytes with abnormal morphologic and physiologic properties. Specifically, melanocytes from vitiligo patients cultured under limiting conditions express a lag-time in growth and are difficult to subculture. Moreover, melanocytes from normally pigmented regions of vitiligo patients display melanosome compartmentalization and dilated ER profiles in vitro as well as in situ $(7,8)$. It is likely that the abnormality within the VIT1 gene may lead to both dilated ER and melanocyte dysfunction.

Herein, we provide the first evidence that VIT1/FBXO11 has an impact on the proliferation and apoptosis of melanocytes and the functional export of tyrosinase from ER. The results will shed light on the potential role of VIT1/FBXO11 in the pathogenesis of vitiligo.

\section{Materials and methods}

Cell culture and transfection. Primary culture of human melanocytes was initiated from neonatal foreskins. Fresh skin specimens were washed 3 times with Hanks' balanced salt solution and excess fat was removed. The samples were cut into small pieces and incubated in $0.25 \%$ trypsin solution at $4^{\circ} \mathrm{C}$ overnight. Epidermis was separated from the dermis 
and epidermal cells were suspended and cultured in Ham's F10 nutrient medium with $10 \%$ fetal bovine serum, $85 \mathrm{nM}$ 12-O-tetradecanoylphorbol-13-acetate (TPA), $0.1 \mathrm{mM}$ 3-isobutyl-1-methylxanthine (IBMX), $2.5 \mathrm{nM}$ cholera toxin (CT), and $100 \mu \mathrm{g} / \mathrm{ml}$ geneticin. B10BR mouse melanocytes were cultured in Ham's F12 supplemented with $10 \%$ horse serum, $50 \mathrm{ng} / \mathrm{ml}$ phorbol 12-myristate 13 -acetate (TPA) and $1 \%$ penicillin/ streptomycin $(9,10)$. All cells were grown at $37^{\circ} \mathrm{C}$ with $5 \% \mathrm{CO}_{2}$.

The polymerase chain reaction (PCR) product of FBXO11 gene was inserted into the HindIII/BamHI digested p3XFLAGCMV-10 plasmid to construct $F B X O 11$ gene overexpressed vector p3XF-P120. siRNAs against FBXO11 gene were generated by Invitrogen, USA. Preliminary experiments showed that only two siRNAs exhibited significant suppression (6). The sequences of siRNAs are 5'-AGA UCU UGU UCU CUA UGA ACU GUC C-3' and 5'-GGA CAG UUC AUA GAG AAC AAG AUC U-3'. According to the manufacturer's recommended protocol, cells were transfected with lipofectamine $^{\mathrm{TM}}$ 2000. Transfection efficiency was determined with routine RT-PCR and Western blotting.

RT-PCR and Western blotting. RNA was isolated from the $\mathrm{B} 10 \mathrm{BR}$ cells at $60 \mathrm{~h}$ post-transfection. Total RNA was prepared using TRIzol reagent (Invitrogen) and stored at $-80^{\circ} \mathrm{C}$. For semi-quantitative RT-PCR, total RNA was reversely transcribed to investigate relative expression of $F B X O 11$ gene. Primers used for amplifying $F B X O 11$ gene were 5'-CAT TAAGAAGTGCCATCA-3' and 5'-GTTACAATGGCAG GACTT-3' (the size of fragment is $298 \mathrm{bp}$ ). As an internal control, actin gene was determined by primers: 5'-CGA GGCCCAGAGCAAGAG-3' and 5'-TAGGGTTCAGGG GGGCC-3' (the size of fragment is $170 \mathrm{bp}$ ). RT-PCR kit (Promega, USA) was used to generate target fragment, and the PCR products were size fractionated on a $2 \%$ agarose gel.

For total protein extraction, B10BR cells were scraped in cold D-PBS and collected by centrifugation, and subsequently lysed in RIPA buffer $\left(10 \mathrm{mM} \mathrm{NaPO}_{4}, \mathrm{pH} 7.4 / 300 \mathrm{mM}\right.$ $\mathrm{NaCl} / 0.1 \%$ SDS/1\% Nonidet P-40/1\% deoxycholic acid/ 2 mM EDTA) with protease inhibitors (Pierce, USA). For Western blotting, $80 \mu \mathrm{g}$ of proteins were separated by SDSPAGE, and were electrophoretically transferred to nitrocellulose membranes. Following blockage by $5 \%$ nonfat dry milk in TBS with $0.1 \%$ Tween-20, the membranes were incubated with primary antibodies at 1:1000 dilution overnight at $4^{\circ} \mathrm{C}$. Applicable secondary antibodies were used to detect the appropriate primary antibodies. Immunoblotted membranes were developed by using the ECL Western blotting system (Thermo Scientific Biotechnology, USA). Quantification of appropriate bands on Western images was done using the quantification software of digital imaging system $\left(\right.$ ChemiDoc $^{\text {TM }}$ XRS+, Bio-Rad, USA).

Transwell cell migration assay. The lower surface of nucleopore filters with $8-\mu \mathrm{m}$ pore size (Corning, USA) was precoated with fibronectin and dried at room temperature. B10BR cells were harvested and resuspended in serum-free medium (1\%BSA). Cell suspension $(100 \mu 1)\left(1 \times 10^{5}\right.$ cells $)$ and $600 \mu \mathrm{l}$ of integrated medium were added into the upper and lower chamber, respectively. After $20 \mathrm{~h}$ of incubation, the cells adhered to lower layer were stained by crystal violet, and then crystal violet was eluted with $33 \%$ acetic acid. The absorbance of the elution solution at $570 \mathrm{~nm}$ was detected with the microplate spectrophotometer (SoftMax ${ }^{\circledR}$ Pro5, Molecular Devices, USA).

Zymography. Zymography was performed in 10\% SDSpolyacrylamide gel electrophoresis containing $0.1 \%$ gelatin. Supernatants derived from B10BR was mixed with undenatured sample buffer and subjected to electrophoresis without boiling. According to the protocol of zymography kit, activity of matrix metalloproteinase MMP2 and MMP9 was detected. The gels were recorded and analyzed on a digital imaging system (ChemiDoc XRS+, Bio-Rad).

MTT dye assay. B10BR cells were seeded at $1 \times 10^{4}$ per well in 96-well flat-bottom plates 1 day before transfection. After transfection for 24,48 and $72 \mathrm{~h}$, followed by the addition of $10 \mu 1$ of $10 \mathrm{mg} / \mathrm{ml}$ MTT to each well, cells were incubated for another $4 \mathrm{~h}$, and $100 \mu \mathrm{l}$ of DMSO was added and then lysed for $15 \mathrm{~min}$. The absorbance value at $490 \mathrm{~nm}$ was measured with the microplate spectrophotometer (SoftMax Pro5, Molecular Devices).

FACS for apoptosis and cell cycle analysis. B10BR cells were seeded at $7.5 \times 10^{4}$ per well in 6 -well plates and transfected as described above. At $48 \mathrm{~h}$ post-transfection, cells were harvested and stained with FITC-labeled Annexin V and propidium iodide (Sigma, USA) to explore apoptosis on the flow cytometer (FACS Calibur, BD, USA). In addition, the cells were harvested, fixed in $70 \%$ ethanol for $12 \mathrm{~h}$ at $4^{\circ} \mathrm{C}$, and stained with PI in PBS containing RNase (Roche, Switzerland) for the cell cycle analysis.

Confocal laser scanning microscopy (CLSM). To examine co-localization of tyrosinase and calreticulin, human epidermal melanocytes were grown in 6-well plates containing glass coverslips, and were either seeded untreated or transfected with plasmid p3XF-P120 or siRNA. At $60 \mathrm{~h}$ post-transfection, all cells were fixed with $4 \%$ formaldehyde in PBS for $30 \mathrm{~min}$. Anti-tyrosinase and anti-calreticulin polyclonal antibodies (Abcam Biotechnology, USA) were used at $1 \mu \mathrm{g} / 100 \mu \mathrm{l}$ in buffer $(0.5 \%$ BSA in PBS) and incubated with the coverslips for $1 \mathrm{~h}$. The coverslips were then incubated in secondary antibodies conjugated to $\mathrm{Cy} 3$ or FITC (Beyotime, China) at 1:200. The coverslips were washed $(3 \times 5 \mathrm{~min})$ in PBS. The slides were mounted using a mounting medium and observed with Confocal laser scanning microscope (TCS SP2, Leica, Germany).

Electron microscopy. After treatment, B10BR cells were collected by trypsin-EDTA (Gibco, USA) and were fixed with $2.5 \%$ glutaraldehyde in $0.1 \mathrm{M}$ phosphate buffer at $4^{\circ} \mathrm{C}$ overnight. Then post-fixed with $1 \% \mathrm{OsO}_{4}$ in $0.1 \mathrm{M}$ phosphate buffer at $4^{\circ} \mathrm{C}$ for $1 \mathrm{~h}$, the cells were embedded in $5 \%$ agarose which were then cut into $2-3 \mathrm{~mm}^{2}$ blocks, dehydrated in a graded series of ethanol and embedded in epoxy resin. Ultrathin sections were stained with uranyl acetate and lead citrate and were observed with an electron microscope (JEM1230, Tokyo, Japan). 


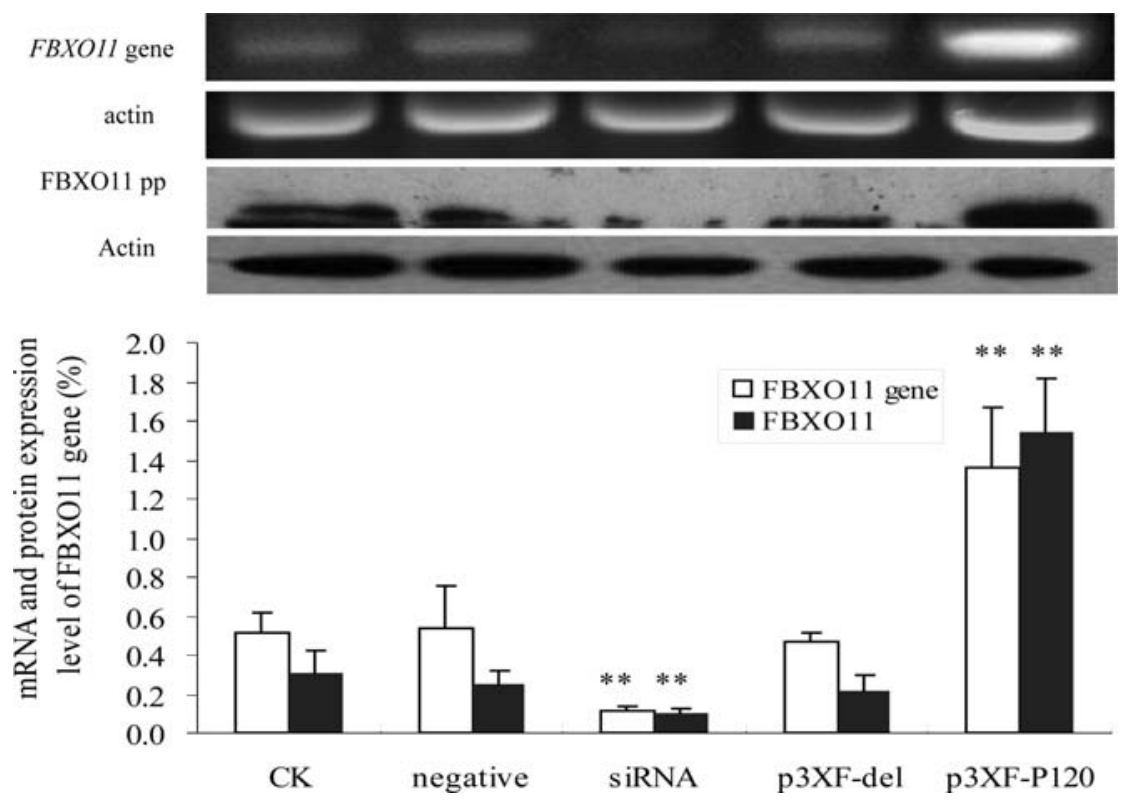

Figure 1. Relative amount of mRNA and protein expression of $F B X O 11$ gene at $24 \mathrm{~h}$ post-transfection. Cells were cultured and transfected with various reagents, and mRNA and proteins expression of FBXO11 gene were detected by RT-PCR and Western blotting analysis. ${ }^{* *} \mathrm{P}<0.01$.

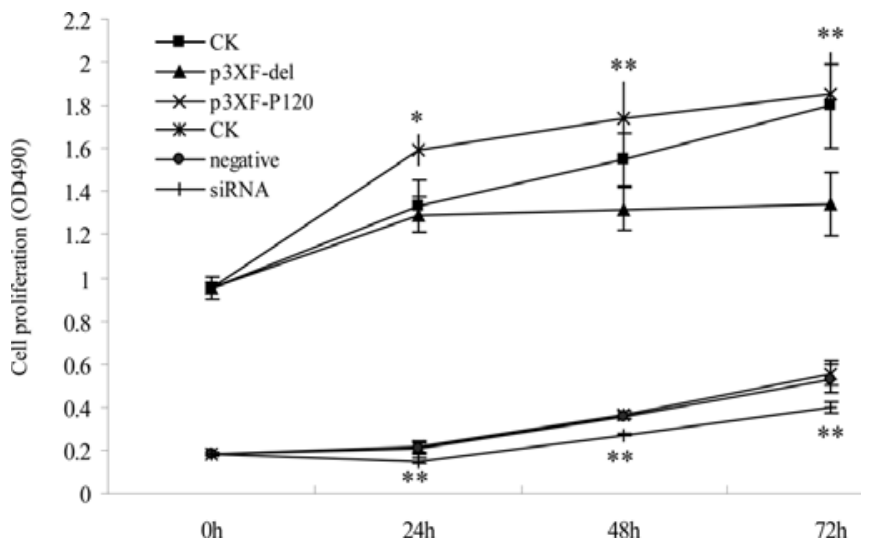

Figure 2. Effect of FBXO11 gene expression on cell proliferation. Cells were cultured and transfected with various reagents and cell proliferation was measured by MTT assay. ${ }^{*} \mathrm{P}<0.05,{ }^{* *} \mathrm{P}<0.01$.

Statistical analysis. All experiments were repeated at least 3 times. Results are presented as mean \pm SEM. Experimental groups were compared by one-way ANOVA analysis of SPSS 13.0. Mean differences were considered significant and extremely significant when they were $\mathrm{P}<0.05$ and $\mathrm{P}<0.01$, respectively.

\section{Results}

Expression of FBXO11 gene after transfection. To study the function of FBXO11 in melanocytes, B10BR cells were either transfected with $F B X O 11$ gene overexpressed vector p3XFP120 (p3XF-del as a plasmid control) or FBXO11 siRNA (negative siRNA as a siRNA control). The untreated B10BR cells (named CK group) were used as general control. Transfection efficiency was estimated by RT-PCR and Western blotting analysis at $24 \mathrm{~h}$ post-transfection. As shown in Fig. 1, compared to the CK group, markedly increased mRNA and protein expression of $F B X O 11$ gene was found in the p3XF-P120 group $(\mathrm{P}<0.01)$, and significantly decreased mRNA and protein expression of FBXO11 gene was observed in the siRNA group $(\mathrm{P}<0.01)$. As expected, no significant difference was found among the $\mathrm{CK}$, negative siRNA and p3XF-del groups regarding both mRNA and protein expression of FBXO11 gene.

Effect of FBXO11 knockdown on cell proliferation. To test the effect of FBXO11 on melanocyte cell proliferation, MTT assay was applied at 24, 48 and $72 \mathrm{~h}$ post-transfection. As shown in Fig. 2, compared to indicated control groups, knockdown of FBXO11 gene greatly inhibited cell proliferation $(\mathrm{P}<0.01)$, while the overexpression of $F B X O 11$ gene in the p3XF-P120 group remarkably increased cell proliferation $(\mathrm{P}<0.01$ or $\mathrm{P}<0.05)$. These data suggest that FBXO11 may contribute to the regulation of melanocyte proliferation.

Effect of FBXO11 knockdown on cellular apoptosis. To further investigate the role of FBXO11, FACS analysis was applied for cell apoptosis detection. As shown in Table I, the early apoptotic rate of cells transfected with siRNA was increased from $10.24 \pm 1.00 \%$ to $37.34 \pm 3.26 \%$, the late apoptotic rate was decreased from $22.22 \pm 2.16 \%$ to $0.25 \pm 0.02 \%$, and both rates were significantly different than the negative cells $(\mathrm{P}<0.01)$. In contrast, the early apoptosis rate in the p3XF-P120 group was decreased from $14.58 \pm 1.49 \%$ to $8.43 \pm 0.86 \%(\mathrm{P}<0.01)$ and the viable cell rate was increased from $52.13 \pm 5.34 \%$ to $71.24 \pm 7.31 \%(\mathrm{P}<0.01)$ versus with p3XF-del cells.

Effect of FBXO11 knockdown on cell cycle. To further study the role of FBXO11 gene in cell cycle, the phase distribution 
Table I. Effect of FBXO11 gene expression on cell apoptosis.

\begin{tabular}{lccccr}
\hline & \multicolumn{5}{c}{ Cell apoptosis $(\%)$} \\
\cline { 2 - 6 } Signal & CK & Negative & siRNA & p3XF-del & p3XF-P120 \\
\hline LL & $82.15 \pm 7.37$ & $66.93 \pm 6.51$ & $62.39 \pm 5.44$ & $52.13 \pm 5.34$ & $71.24 \pm 7.31^{\mathrm{b}}$ \\
LR & $4.43 \pm 0.40$ & $10.24 \pm 1.00$ & $37.3 \pm 3.26^{\mathrm{b}}$ & $14.58 \pm 1.49$ & $8.43 \pm 0.86^{\mathrm{b}}$ \\
UR & $12.74 \pm 1.14$ & $22.22 \pm 2.16$ & $0.25 \pm 0.02^{\mathrm{b}}$ & $27.12 \pm 2.78$ & $20.03 \pm 2.06^{\mathrm{a}}$ \\
\hline
\end{tabular}

${ }^{\mathrm{a}} \mathrm{P}<0.05 ;{ }^{\mathrm{b}} \mathrm{P}<0.01$ vs. untreated. $\mathrm{LL}, \mathrm{PI}^{-} / \mathrm{AV}^{-}$viable cell; $\mathrm{LR}, \mathrm{PI}^{-} / \mathrm{AV}^{+}$early apoptosis; $\mathrm{UR} \mathrm{PI}^{+} / \mathrm{AV}^{-}$late apoptosis.
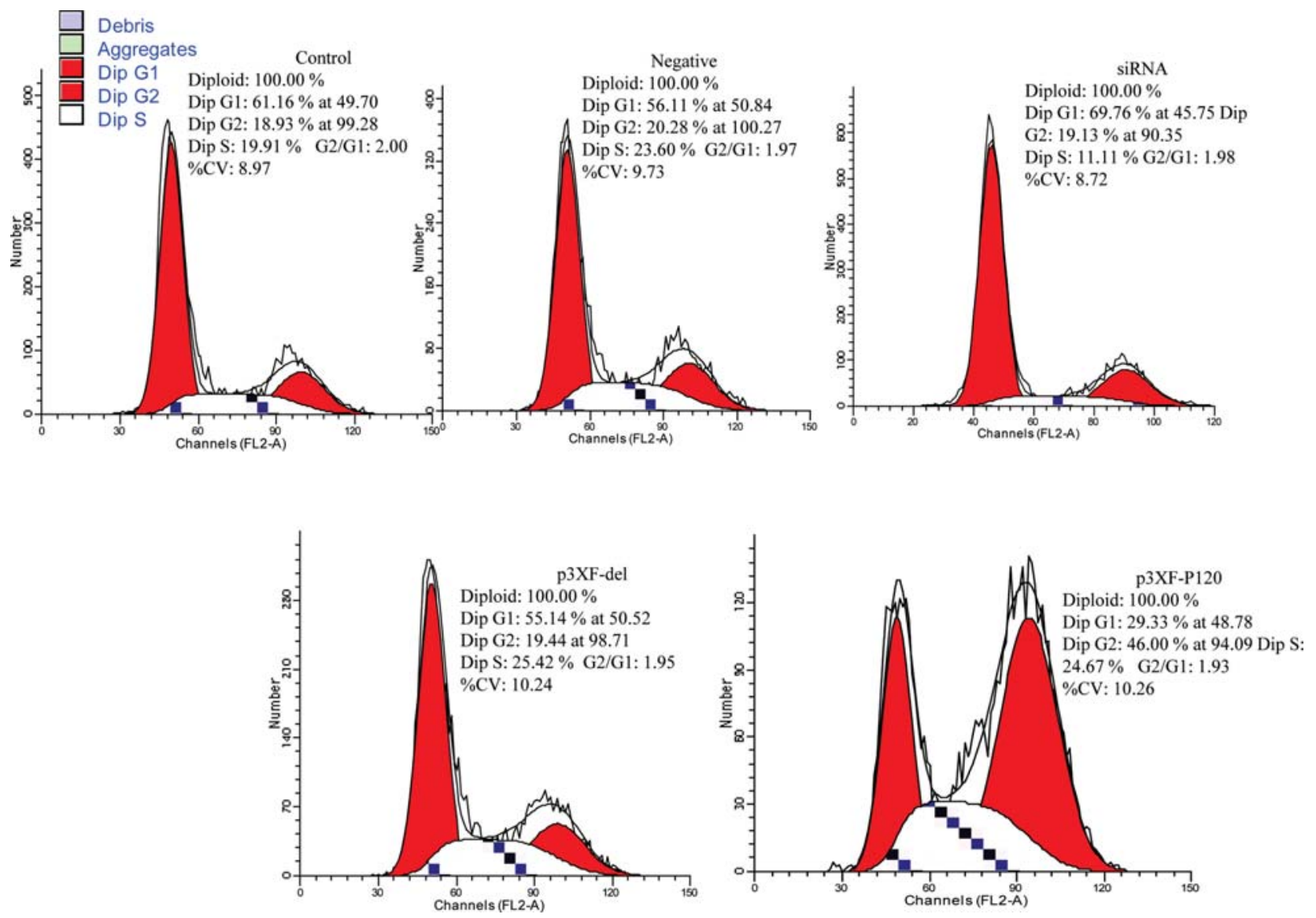

Figure 3. Effect of FBXO11 gene expression on cell cycle. Cells were cultured and transfected with various reagents. Cell cycle analysis was by FACS.

of the cell cycle was analyzed by FACS (Fig. 3). Compared to the negative control group, cell cycle distribution was changed dramatically in the siRNA group, the number of cells in G0/G1 phase was increased from $56.11 \pm 5.46 \%$ to $69.76 \pm 6.08 \%(\mathrm{P}<0.05)$, while the number of cells in $\mathrm{S}$ phase was decreased from $23.60 \pm 2.30 \%$ to $11.11 \pm 0.97 \%(\mathrm{P}<0.01)$. Relative to the p3XF-del group, cells in G0/G1 phase in the p3XF-P120 group were reduced from $55.14 \pm 5.65 \%$ to $29.33 \pm 3.01 \%(\mathrm{P}<0.01)$. In contrast, there were no notable differences in the cell cycle distribution among the $\mathrm{CK}$, the negative and the $\mathrm{p} 3 \mathrm{XF}-\mathrm{del}$ groups $(\mathrm{P}>0.05)$.

Effect of FBXO11 knockdown on cell migration. To further study the biological functions of FBXO11, transwell assay was applied for melanocyte migration. Cells adhered to lower-layer of transwell membrane were stained after $24 \mathrm{~h}$ of incubation and the absorbance was read (Fig. 4). Relative to the CK group $(0.350 \pm 0.054)$, no significant difference of cell migration was found among the p3XF-P120 group $(0.290 \pm 0.034)$, the siRNA group $(0.380 \pm 0.045)$, the negative group $(0.364 \pm 0.049)$ and the p3XF-del group $(0.312 \pm 0.033)$ $(\mathrm{P}>0.05)$.

Effect of FBXO11 on MMP expression. B10BR cells were maintained in serum-free medium for $16 \mathrm{~h}$ after $24 \mathrm{~h}$ posttransfection, and the supernatant was collected to detect the activity of MMP2 and MMP9 with a zymography kit. As shown in Fig. 5, two bright bands were present at the position 


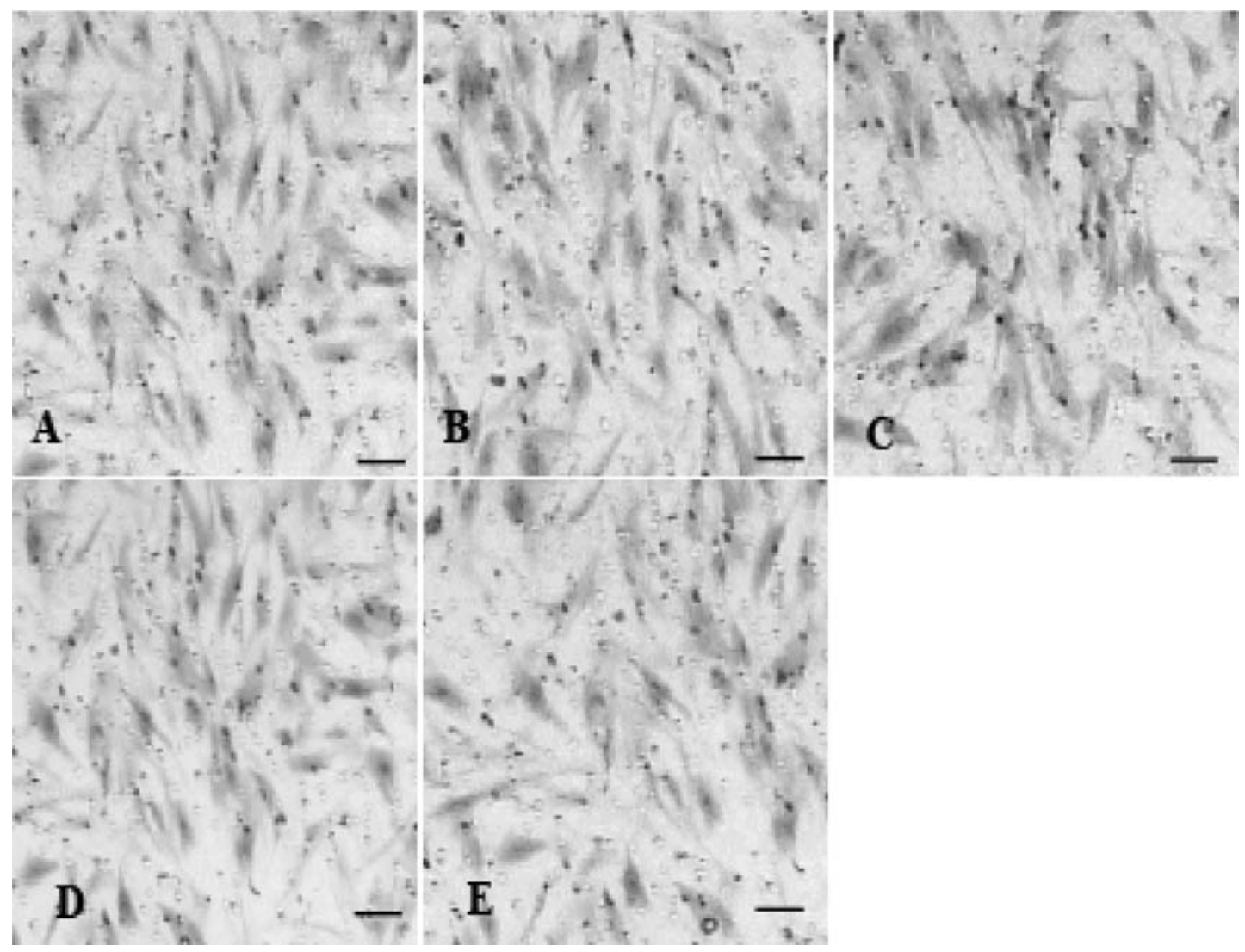

Figure 4. Transwell analysis of cell migration after transfection. Cells were cultured and transfected with various reagents. Cell migration was measured by transwell assay (Crystal violet, x400). (A) CK; (B) negative; (C) siRNA; (D) p3XF-del; (E) p3XF-P120.

CK

negative

siRNA

p3XF-del

p3XF-P120
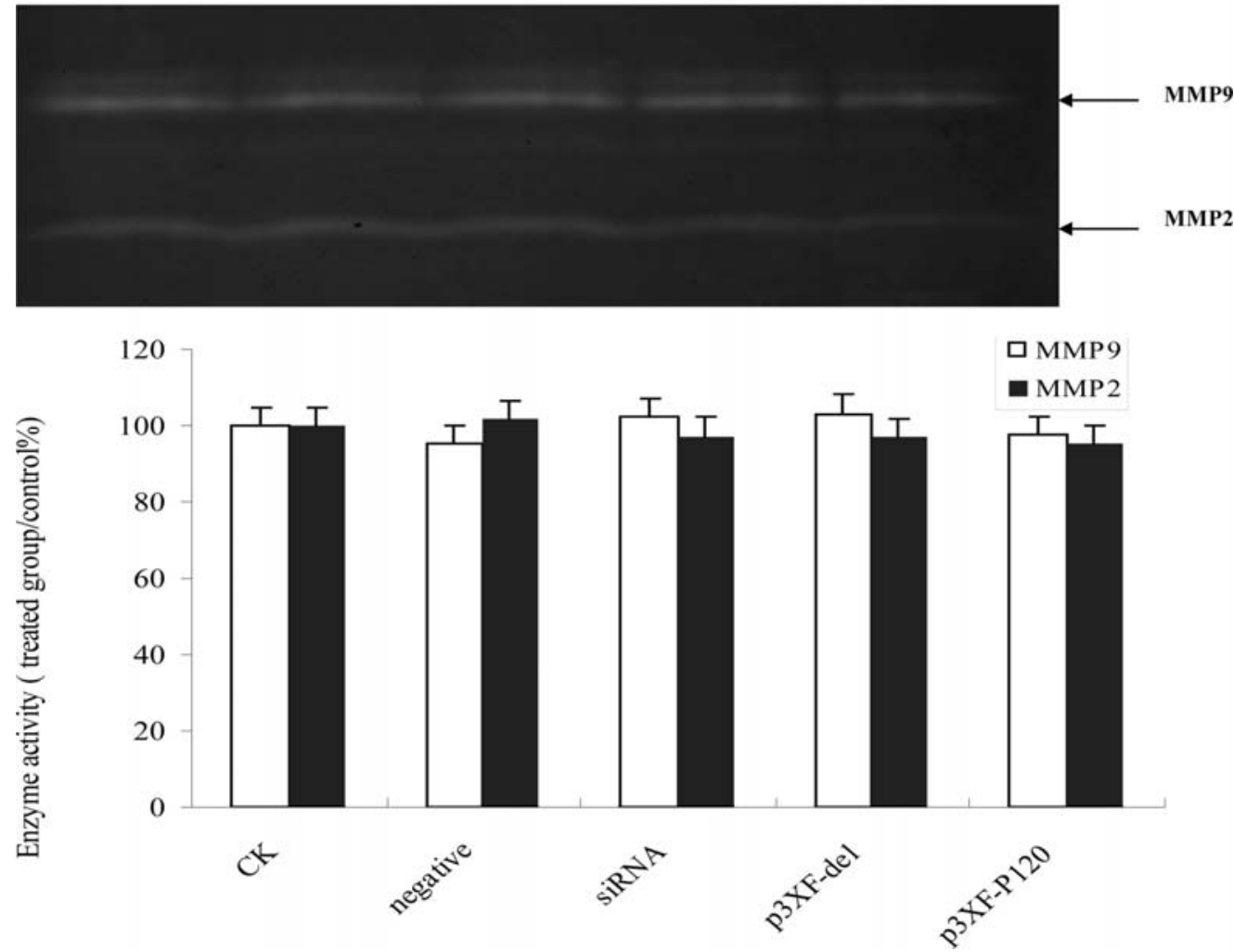

Figure 5. Zymography for matrix metalloproteinase MMP2 and MMP9. Cells were cultured and transfected with various reagents. The conditioned media were collected for zymography assay for MMP9 and 2 activity. 

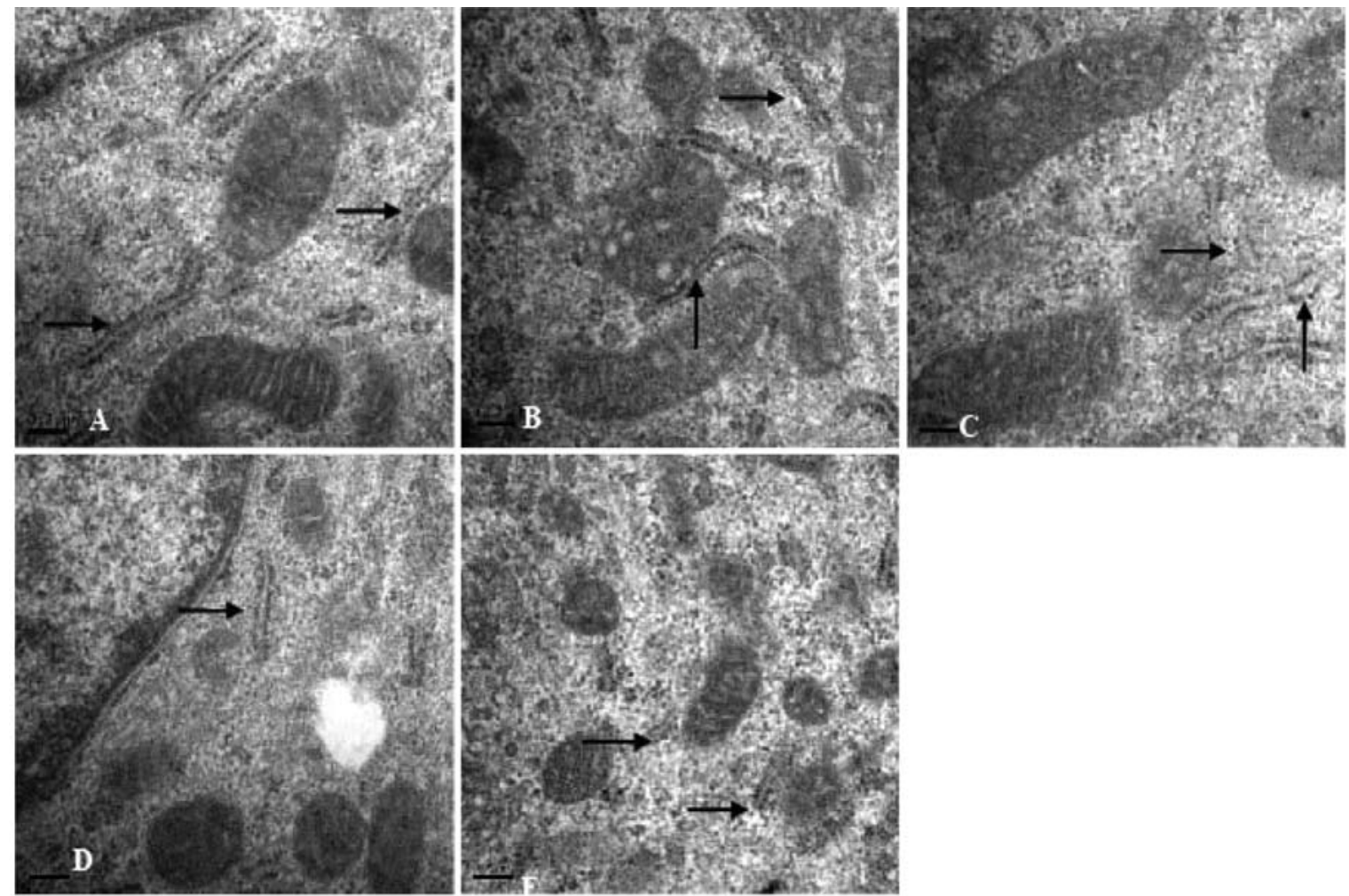

Figure 6. FBXO11 gene impacts on the modality of endoplasmic reticulum of B10BR melanocytes. Cells cultured and transfected with various reagents and were prepared for electron microscopic analysis. (A) CK; (B) negative; (C) siRNA; (D) p3XF-del; (E) p3XF-P120. Arrowheads point to ER. Magnification is $\mathrm{x} 40000$, the bar represents $0.2 \mu \mathrm{m}$.

of MMP2 and MMP9, and no significant difference was observed among all the groups $(\mathrm{P}>0.05)$.

Effect of FBXO11 knockdown on ER configuration. Electron microscopic analysis was carried out to investigate the impact of FBXO11 gene on ER configuration in B10BR cells. As shown in Fig. 6, dilated ER was observed in the siRNA group. In contrast, ER configuration in the other four groups was shown to be normal. The data suggest that the FBXO11 gene is involved in ER configuration.

Co-localization of tyrosinase and calreticulin. Confocal laser scanning microscopy was performed to assess the colocalization of tyrosinase and calreticulin. In the CK, negative, p3XF-del and p3XF-P120 groups, distribution of tyrosinase fluorescence (green) was pronounced in ER marked by calreticulin (red fluorescence as ER marker). However, in the siRNA group, a large portion of tyrosinase fluorescence overlapped with the fluorescence of ER marker protein (Fig. 7). This data suggests that silencing of FBXO11 gene interferes with the functional export of tyrosinase from ER. To further examine the expression of calreticulin (ER marker protein) and tyrosinase (a key protein in melanocyte function), Western blotting analysis was applied. The results (Fig. 8) showed no significant difference of calreticulin expression among the groups. Tyrosinase expression in the p3XF-P120 group was higher than that in the $\mathrm{CK}$ group, the negative group and the p3XF-del group $(\mathrm{P}<0.01)$, which suggests that FBXO11 promotes tyrosinase expression. However, in the siRNA group, tyrosinase expression was shown to be extraordinarily high compared with the other four groups $(\mathrm{P}<0.01)$. These data suggest that abnormal export of tyrosinase from ER leads to obstruction of degradation, and further accumulation.

\section{Discussion}

Our previous study indicated that VIT1 gene in Chinese vitiligo patients without introns is in fact a previously identified gene called $F B X O 11$. Our preliminary study was mainly focused on the effects of FBXO11 siRNA on melanocyte apoptosis and ultrastructure. We further investigated the effects of inhibition and overexpression of $F B X O 11$ gene on the biological behavior of melanocytes, and on the export of tyrosinase from ER.

We designed and synthesized siRNA and expression vectors for the $F B X O 11$ gene, which were stably transfected into B10BR cell line and human epidermal melanocytes. Compared to the control group, the mRNA and protein expression levels of $F B X O 11$ gene were significantly reduced in the siRNA group, while those in the p3XF-P120 group were markedly increased. Both decreased proliferation rate and increased apoptosis rate were found in the siRNA group. On the contrary, there was increased cell proliferation and reduced apoptosis in the p3XF-P120 group. These results suggest that the $F B X O 11$ gene is involved in cell proliferation and apoptosis in melanocytes, which may be in accordance with the depletion of melanocyte populations in vitiligo patients.

In addition to the suppression of apoptosis, those cells are also characterized by deregulated cell proliferation, which is generally associated with accelerated G1/S and G2/M cell 


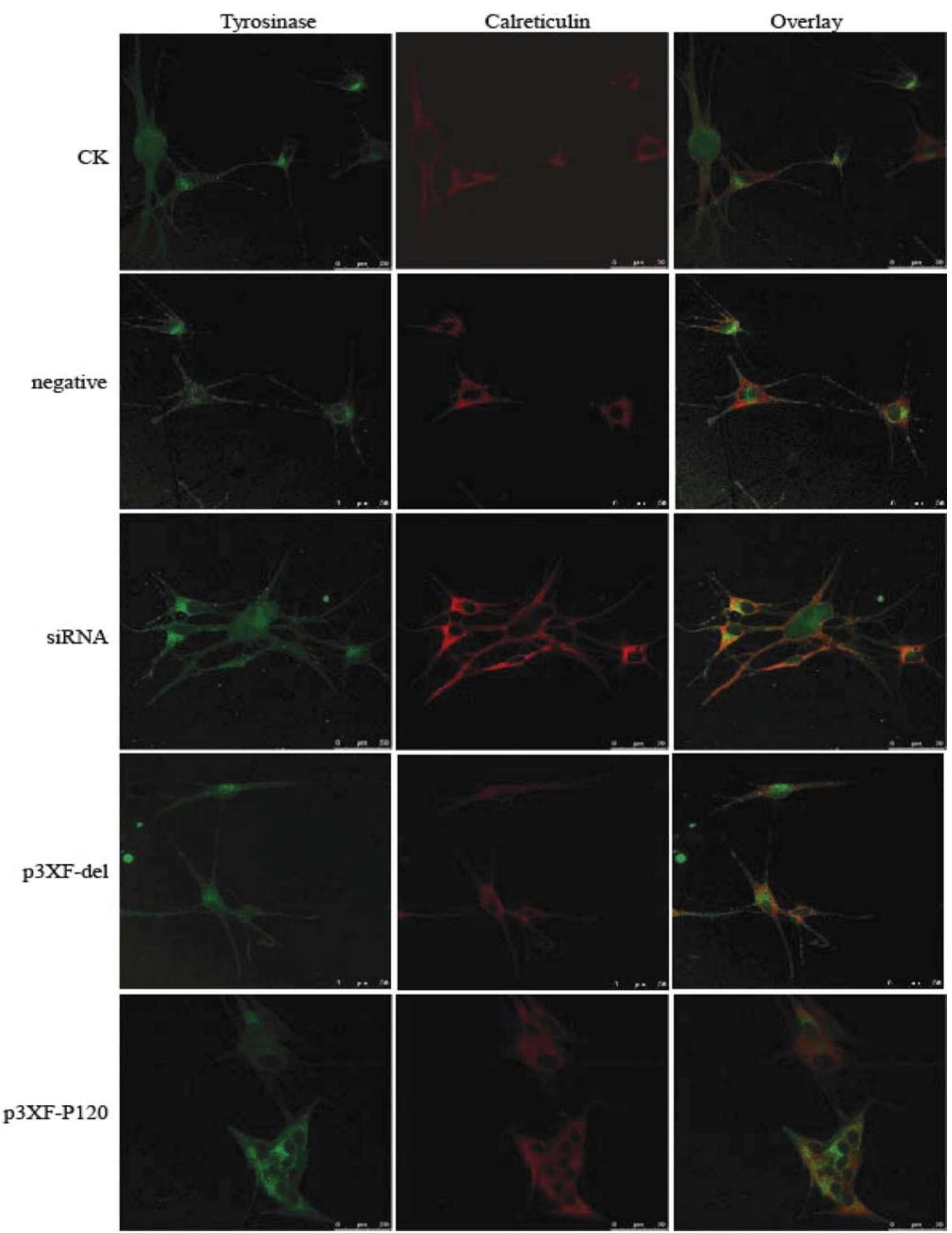

Figure 7. Confocal microscopic observation of tyrosinase and calreticulin in cells. Cells were cultured, transfected with various reagents, and prepared for confocal microscopic analysis. Magnification is x630, and the bar represents $50 \mu \mathrm{m}$.

cycle transitions (11). Enhanced transit from G1 to S phase correlates with increased cell proliferation. G1 arrest of the cell lines analyzed herein reflects an inhibition of cell proliferation. The selective degradation of many proteins is carried out by ubiquitin-mediated proteolytic system (12). The ubiquitin system plays important roles in cell cycle control 


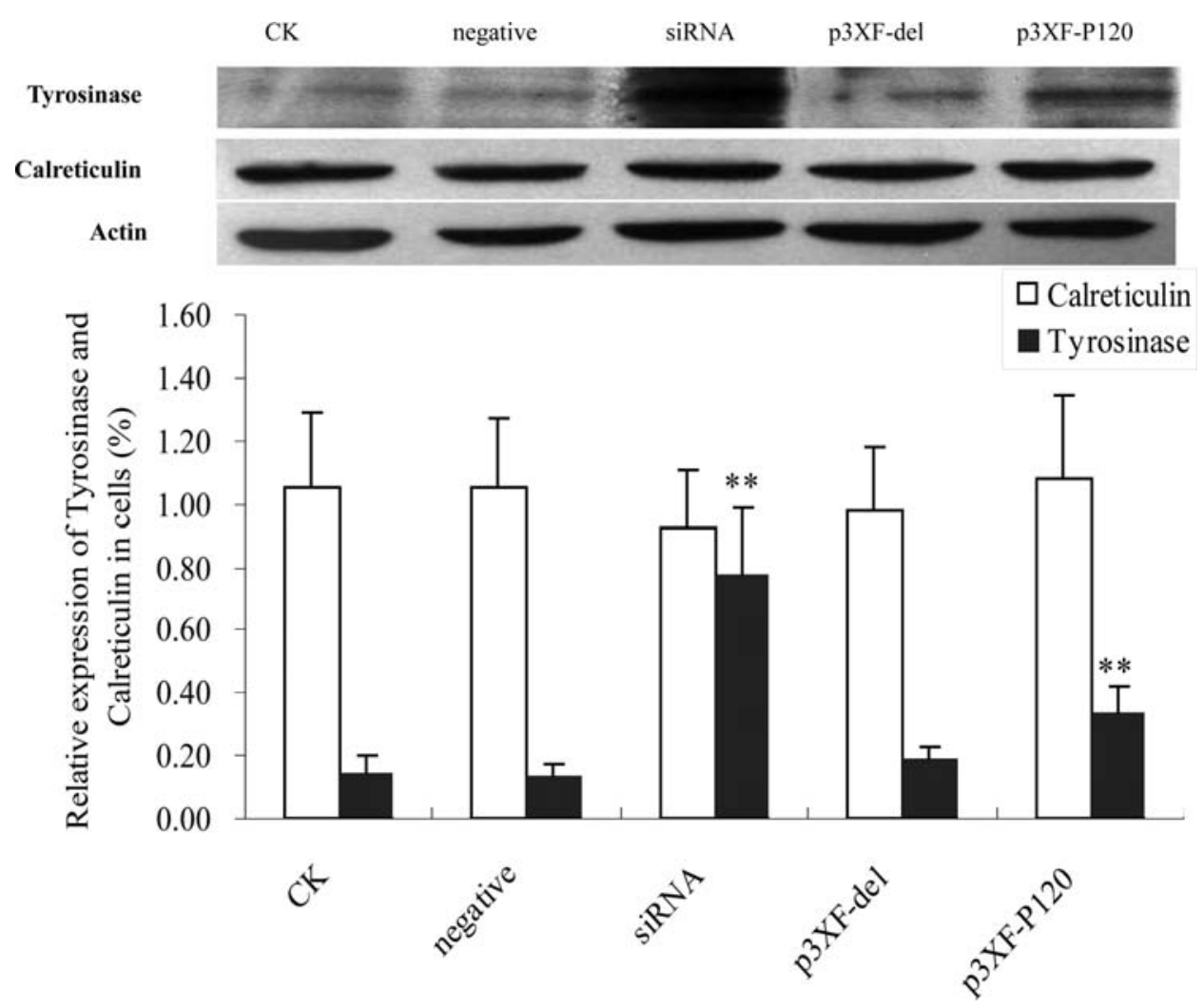

Figure 8. Expression of tyrosinase and calreticulin. Cells were cultured and transfected with various reagents, and the expression of tyrosinase and calreticulin was measured by Western blot analysis. ${ }^{* *} \mathrm{P}<0.01$.

$(13,14)$, and SCF connects cell cycle regulators to the ubiquitin proteolysis machinery (15).

We hypothesize that FBXO11 gene is associated with cell cycle arrest at the G0/G1 or G2 phases, which reduces the apoptosis rate and results in higher cell proliferation. In this study, the accumulation of cells in G0/G1 phase and the reduction of cells in $\mathrm{S}$ phase treated with siRNA for FBXO11 suggest that the $F B X O 11$ gene is associated with decreased ability to progress from G0/G1 phase to $S$ phase, eventually leading to the inhibition of proliferation. In contrast, we observed a reduced number of cells in $\mathrm{G} 0 / \mathrm{G} 1$ phase in the p3XF-P120 group.

In addition, in this study, the FBXO11 gene was inhibited and overexpressed in B10BR cells to investigate its potential role in the migration of melanocytes. The data showed that no significant difference was found among all groups. Similarly, no significantly difference on the activity of MMP2 and MMP9 was shown among the five groups. These results suggest that the FBXO11 gene is not involved in the regulation of MMP2 and MMP9 activity, or melanocyte migration.

Tyrosinase is the core enzyme catalyzing melanogenesis in melanocytes, and is highly important in melanin formation. Abnormalities in tyrosinase post-translational processing have been revealed in several depigmentation diseases. Stagnation of tyrosinase in ER was relevant to the phenotype of pigment loss in melanoma. From golgi to melanosome, dysfunctional transportation of tyrosinase leads to different diseases, such as generalized albinism types 2 and 4 and Hermansky-Pudlak syndrome $(16,17)$. F-box in FBXO11 gene was shown to be neddylated, and its modification was shown to be important for the ubiquitin ligase function of some SCF complexes $(4,18)$.

SCF induces protein degradation, and reduced SCF causes dilated ER with a result of deficiency of melanogenesis. The normal skin of vitiligo patients showed abnormal ultrastructure of ER (8), in which some melanogenesis-related proteins with disordered processing or/and transportation were accumulated, but precisely what proteins remain unclear (5). Considering the potential role of FBXO11 in ER function, we used electron microscopy. We found that in all groups except for the siRNA group, ER configuration was normal, while in the siRNA group, dilated ER was prominent. This data suggest that one or more proteins were accumulated in the ER when the FBXO11 gene was inhibited. However, the identity of these proteins remains unclear. Since the export of tyrosinase is important for the normal function of melanocytes, whether tyrosinase was accumulated in melanocytes from vitiligo patients needs to be further assessed. Further investigation regarding the effect of FBXO11 gene on tyrosinase export was studied by CLSM to detect the colocalization of tyrosinase and calreticulin.

Apparently, in the siRNA group, most of tyrosinase was localized in ER, but it was largely excluded from the ER marked by calreticulin in the other four groups. This result suggests that tyrosinase could not be exported from ER normally when $F B X O 11$ gene was inhibited. We then simultaneously measured the expression level of tyrosinase and calreticulin by Western blotting. The results showed that 
overexpression of $F B X O 11$ gene could induce tyrosinase expression. Expression of tyrosinase in the siRNA group was remarkably higher than that in other four groups. These data suggest that as FBXO11 gene is inhibited, export of tyrosinase from ER is disabled, potentially leading to the accumulation of tyrosinase within the ER. Our confocal microscopic data support this notion.

In conclusion, our data show that FBXO11 is involved in a variety of cellular processes in melanocytes, including regulation of apoptosis and proliferation, and export of tyrosinase from ER. Further understanding of the important roles of FBXO11 in abnormal melanocytes in vitiligo patients would provide targets for the clinical management of vitiligo.

\section{Acknowledgements}

We thank Dekuang Zhao, Seth Wander (Cancer Biology Program, University of Miami, FL, USA) for critical reading of the manuscript. This study was supported partly by grants from the National Natural Science Foundation of China (No. 30671896, 30872280) and the Natural Science Foundation of Zhejiang Province (Z206607) and a grant from NIH (P20 RR016457 from INBRE Program of the National Center for Research Resources).

\section{References}

1. Howitz J, Brodthagen H, Schwartz $M$ and Thomsen $K$ : Prevalence of vitiligo. Epidemiological survey on the Isle of Bornholm, Denmark. Arch Dermatol 113: 47-52, 1977.

2. Alkhateeb A, Stetler GL, Old W, et al: Mapping of an autoimmunity susceptibility locus (AIS1) to chromosome $1 \mathrm{p} 31.3$ p32.2. Hum Mol Genet 11: 661-667, 2002.

3. Fain PR, Gowan K, LaBerge GS, et al: A genomewide screen for generalized vitiligo: confirmation of AIS1 on chromosome $1 \mathrm{p} 31$ and evidence for additional susceptibility loci. Am J Hum Genet 72: 1560-1564, 2003

4. Zhang XJ, Liu JB, Gui JP, et al: Characteristics of genetic epidemiology and genetic models for vitiligo. J Am Acad Dermatol 51: 383-390, 2004
5. Le Poole IC, van den Wijngaard RM, Westerhof W, et al: Presence or absence of melanocytes in vitiligo lesions: an immunohistochemical investigation. J Invest Dermatol 100: 816-822, 1993

6. Li Y, Chen F, Lin F, et al: VIT1/FBXO11 knockdown induces morphological alterations and apoptosis in B10BR mouse melanocytes. Int J Mol Med 23: 673-678, 2009.

7. Boissy RE, Beato KE and Nordlund JJ: Dilated rough endoplasmic reticulum and premature death in melanocytes cultured from the vitiligo mouse. Am J Pathol 138: 1511-1525, 1991.

8. Boissy RE, Liu YY, Medrano EE and Nordlund JJ: Structural aberration of the rough endoplasmic reticulum and melanosome compartmentalization in long-term cultures of melanocytes from vitiligo patients. J Invest Dermatol 97: 395-404, 1991

9. Song X, Xu A, Pan W, et al: Nicotinamide attenuates aquaporin 3 overexpression induced by retinoic acid through inhibition of EGFR/ERK in cultured human skin keratinocytes. Int J Mol Med 22: 229-236, 2008.

10. Song X, Xu A, Pan W, et al: Minocycline protects melanocytes against $\mathrm{H}_{2} \mathrm{O}_{2}$-induced cell death via JNK and p38 MAPK pathways. Int J Mol Med 22: 9-16, 2008.

11. Evan GI and Vousden KH: Proliferation, cell cycle and apoptosis in cancer. Nature 411: 342-348, 2001.

12. Jin J, Cardozo T, Lovering RC, et al: Systematic analysis and nomenclature of mammalian F-box proteins. Genes Dev 18: 2573-2580, 2004.

13. King RW, Deshaies RJ, Peters JM and Kirschner MW: How proteolysis drives the cell cycle. Science 274: 1652-1659, 1996.

14. Peters JM: SCF and APC: the Yin and Yang of cell cycle regulated proteolysis. Curr Opin Cell Biol 10: 759-768, 1998

15. Bai C, Sen P, Hofmann K, et al: SKP1 connects cell cycle regulators to the ubiquitin proteolysis machinery through a novel motif, the F-box. Cell 86: 263-274, 1996.

16. Chiang PW, Oiso N, Gautam R, et al: The Hermansky-Pudlak syndrome 1 (HPS1) and HPS4 proteins are components of two complexes, BLOC-3 and BLOC-4, involved in the biogenesis of lysosome-related organelles. J Biol Chem 278: 20332-20337, 2003.

17. Kushimoto T, Valencia JC, Costin GE, et al: The Seiji memorial lecture: the melanosome: an ideal model to study cellular differentiation. Pigment Cell Res 16: 237-244, 2003.

18. Lammer D, Mathias N, Laplaza JM, et al: Modification of yeast Cdc53p by the ubiquitin-related protein rub1p affects function of the SCFCdc4 complex. Genes Dev 12: 914-926, 1998. 